\title{
SOME PARTICULAR INDICES OF EFFECTIVENESS OF UNMANNED AERIAL VEHICLE APPLICATION
}

\author{
Alexei Samkov ${ }^{1}$, Valeriy Silkov ${ }^{2}$ \\ ${ }^{1}$ National Aviation University, Kiev, Ukraine \\ ${ }^{2}$ Central Scientific-Research Institute, Kiev, Ukraine \\ E-mail: ${ }^{1}$ sam_a2006@i.ua (corresponding author)
}

Received 19 March 2012; accepted 20 July 2012

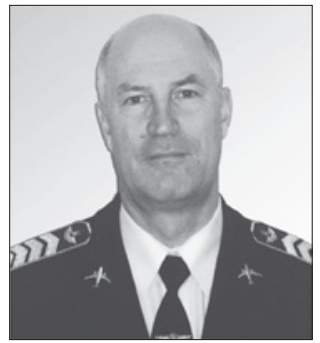

\author{
Alexei SAMKOV, Prof. Dr. Sr. Eng. \\ Education: Kiev Higher Educational Institution of Aviation Military Engineering. \\ Affiliations and functions: head of the Mechanics and Energy Department, National \\ Aviation University. \\ Research interests: technical cybernetics and system methods of aircraft upgrading, UAV \\ design, aerodynamics and dynamics of flight. \\ Publications: over 150 articles. \\ Valeriy SILKOV, Prof. Eng. \\ Education: Kiev Higher Educational Institution of Aviation Military Engineering. \\ Affiliations and functions:senios researcher at the Central Scientific-Research Institute. \\ Research interests: aerodynamics and dynamics of flight, UAV design. \\ Publications: over 170 articles.
}

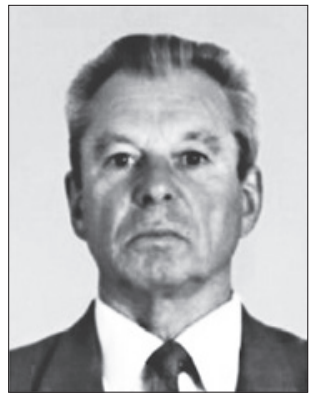

Abstract. In this article, a methodological approach based on a complex indicator (productivity - cost - efficiency) to evaluate the efficiency of using unmanned aeronautical systems is presented as a solution to the problem of searching for objects on the ground. This methodological approach can be used as a substantiation of decisions taken during the act of searching for specific objects and as a comparative evaluation of unmanned aeronautical systems by taking into account their characteristics.

Keywords: UAV, performance, productivity, cost, efficiency.

\section{Introduction}

The tremendous increase in unmanned aerial vehicles (UAV) has raised a variety of questions, in particular methodological ones. In periodicals there is an active discussion about the assessment of the technical level of UAVs and methods to objectively assess their characteristics (Rastopchin 2012; Mitrakovich et al. 2009). It is evident that the problem can be successfully solved only when there is a comprehensive selection of indices (criteria) for comparison. The ability to perform a con- trolled flight without a crew on board gives UAVs significant advantages during their tactical employment. The most widespread application of UAVs is therefore in the military (Mitrakovich et al. 2009). Along with this, every year the range of civil tasks that are fulfilled with the help of unmanned aerial vehicles becomes wider (Goraj 2003). And in the first instance, they are the tasks of surveillance and search. They can significantly differ from each other. For a specified region under surveillance, the requirement of longest duration of flight is set 
for the aircraft in order to have the ability to watch the continuously changing environment. For a search, the main criterion is the rate of receiving information and delivering it to the recipient (for example, information about a ship in distress). In this case, priority is given to the speed of flight and the time of information delivery.

Differences in tasks sets different requirements for aircraft performance and airborne equipment. This article provides the principle of assessing the effectiveness of UAVs according to criteria of capability-cost-agility. The value of each of the components is determined by the purpose of the UAV.

The search for some object (target for military UAV) is often connected with the reconnaissance of some area with the help of airborne electrooptical, radar or infrared systems or other means. In this process, the system of aerial photography covers a strip of land surface of width $B$. Considering the speed of flight $V$ to be constant, during time period $t$ the aircraft passes the distance of $L=$ $V t$ and provides a search of the area $S=B V t$. For accurate calculations, it is necessary to take into account the coefficient of areas overlapping, which is inevitable during practical scanning.

The flight path depends on the search task. If there is no information about the object and it is necessary to discover it in some area $S$, then the flight path can be presented as ordinary scanning (Fig. 1). Such a flight can be programmed and accomplished automatically without interference from an operator.

If there is a need to discover the object on the basis of preliminary information, the flight path can have more complicated profile - horizontal turns, bankings, climb and descent (Fig. 2) (Silkov 2004). The operator can interfere in the control and change the flight mode and profile.

From the given examples, it is evident that in the process of flight there is scanning of some strip of territory under the flight path. These strips can either be tightly joined to each other (Fig. 1) or change randomly

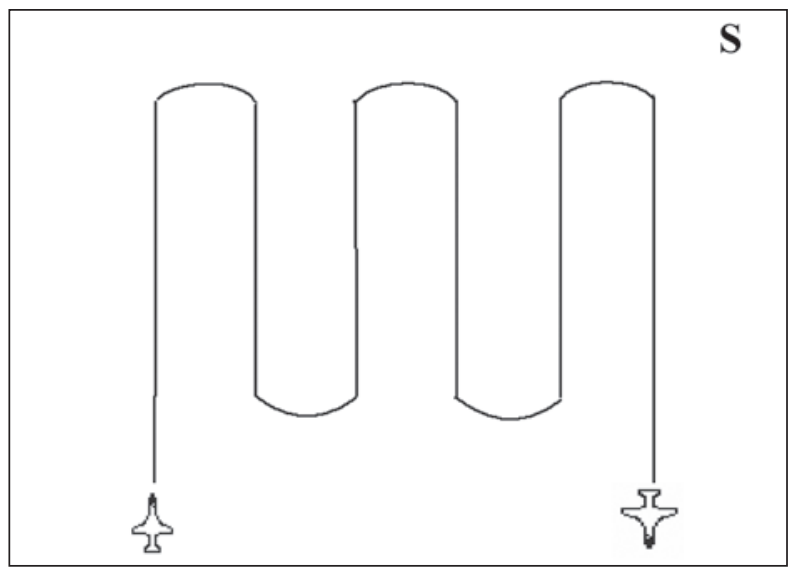

Fig. 1. Scanning of fixed area

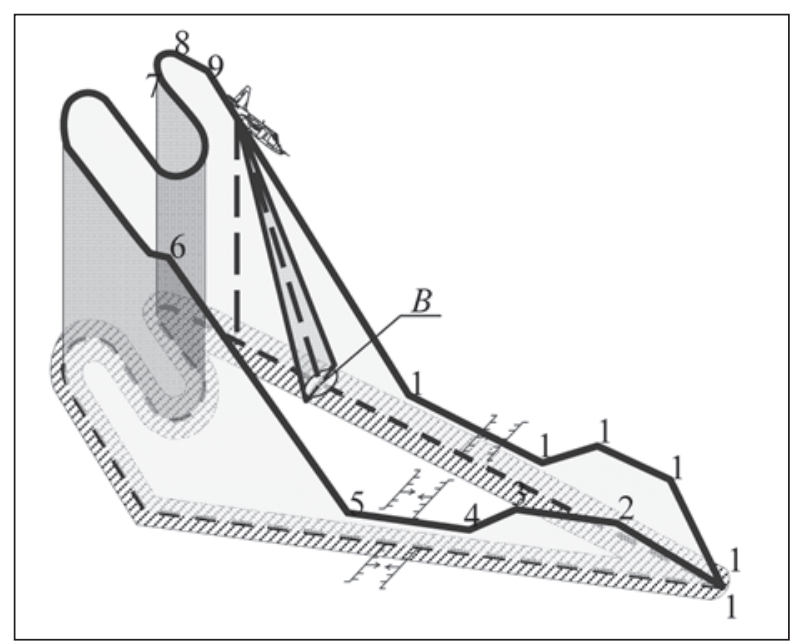

Fig. 2. Space flight path

depending on the flight path (Fig. 2). The area scanned during flight will be equal to

$$
S=B L=B V t,
$$

where $B$ is a territory strip width covered by vehicle equipment, $L$ is the length of the flight route, and $V$ and $t$ are correspondingly the average speed of flight and the duration of surveillance.

Flight altitude is determined by the capabilities of reconnaissance equipment and performance of an aircraft (Mitrakovich et al. 2009). One of the main requirements for a military UAV is the ability to avoid being hit by conventional armaments: gunfire and portable air-defense systems.

Strip width of territory cover $B$ is determined by the angle of camera view $2 \beta$ and flight altitude $H$ (Fig. 3)

$$
B=2 H \operatorname{tg} \beta \text {. }
$$

The highlighted peculiarities require the use of special indices for the assessment of the effectiveness of UAV use. Let us consider them in detail.

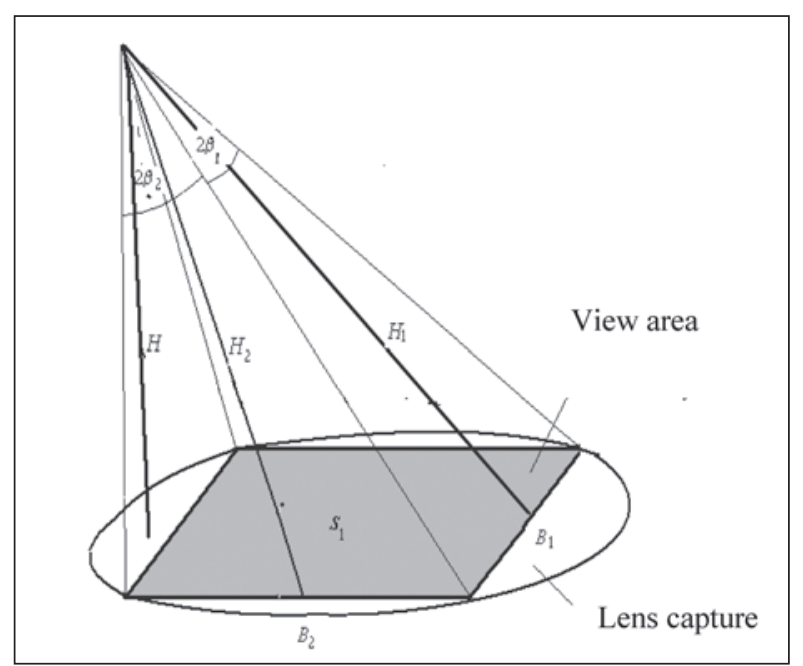

Fig. 3. Object identification platform 


\section{Survey capability (W)}

Some authors estimate that the search capability of unmanned aeronautical reconnaissance systems can quantitatively be assessed by the value of the area surveyed in a unit of time (Rastopchin 2012). Average value of this index can be obtained from formula (1), dividing its right and left parts by time

$$
W=\frac{S}{t}=\frac{B V t}{t}=B V=2 V H \operatorname{tg} \beta,
$$

where $V$ is the average speed of flight, $B$ is the width of the strip covered by the camera view, and $t$ is the duration of reconnaissance. For more accurate assessment, it is necessary to consider the probability of reliable target detection and also the probability of successful passage through the enemy air defence system.

The survey capability shows how fast the observer can investigate the given area. Capability is a complex index because it takes into account aircraft performance (speed and altitude of flight) and also the capacities of surveillance equipment (in particular, the angle of vision area in azimuth).

Obtaining the initial information is carried out in different forms depending on the equipment being installed: either in the form of photographs of the territory with some overlapping of areas and their further interpretation on the ground or by means of continuous surveillance of the territory along the flight route in a real-time scale with the help of video cameras and direct information processing. The second variant is a priority because of its evident advantage, but it is difficult to implement it at a long range. It is also necessary to consider that the rate of information taken and delivered to the ground should not exceed the rate of its processing at the control centre. This is one of the most important problems of obtaining the information in a real-time scale. With limited data processing abilities, there is no need to use an unmanned aeronautical system of high capability to perform such reconnaissance. As an example, it is possible to specify the performance of the Global HAWK unmanned aeronautical system. It can carry out the survey of a strip of territory up to $10 \mathrm{~km}$ in width with linear resolution of $1 \mathrm{~m}$. The capability of the system in such a mode is more than $100,000 \mathrm{~km}^{2}$ daily. In the mode of increased resolution (about $0.5 \mathrm{~m}$ ), surveillance can be carried out over territories of $2 \times 2 \mathrm{~km}$. In this case, it can survey of up to 2000 such territories per day and transmit the information for further processing. Naturally, to process such a volume of information, it is necessary to have several lines or full automatisation.

In case of discrete survey, the volume of information is calculated by the number of frames $n$. It can be determined considering the following assumptions.

Let us presume it is necessary to make a survey of some area $S$ during one flight with the task to search for a certain type of object. For that it is necessary to obtain $n=\kappa_{\mathrm{sq}} S / s_{1}$ frames, where $\kappa_{\mathrm{sq}}=n s_{1} / S$ is a coefficient of the surveyed areas overlapping, $s_{1}$ is an area of a region on which there can be one or several objects that should be discovered and recognised. To decrease the time spent to survey the entire area $S$, the value $s_{1}$ should be greater. But on a large territory, the object can appear to be indistinguishable. Its distinguishability is determined by detail level $d$ (ground resolution). When altitude increases, the level of detail level increases, and the object being searched for can appear to be indistinguishable.

Certain objects will be distinguishable if the level of detail of the survey does not exceed given value $d_{\text {giv }}$ that is $d \leq d_{\text {giv }}$. It is evident that optimum altitude of flight will correspond to the condition $d=d_{\text {giv }}$. This means that the region of survey $s_{1}$ will be maximum, and objects will be distinguishable.

Modern electrooptical systems purposed for surveillance have several angles of vision area: narrow, wide and intermediate. For each of them, there will be certain value of $s_{1}$. Besides, angles of viewing area can be different (Fig. 4) in azimuth $2 \beta_{1}$ and in angles of location $2 \beta_{2}$. As a result, the linear dimensions of objects reproduced by the electrooptical systems will be also different in directions $B_{1}$ and $B_{2}$. Analogically to formula (2), we will have $B_{1}=2 H_{1} \operatorname{tg} \beta_{1}$ and $B_{2}=2 H_{2} \operatorname{tg} \beta_{2}$. Flight altitude $H$ is usually significantly greater than the linear dimensions of the surveyed region, and therefore it is possible to assume $H_{1} \approx H_{2} \approx H$, though at small angles of location, instead of altitude $H_{1}$ it is necessary to take the distance to the object.

Considering all the abovementioned, the area of 'photographed' region will be equal to

$$
s_{1}=B_{1} B_{2}=4 H^{2} \operatorname{tg} \beta_{1} \operatorname{tg} \beta_{2} .
$$

As is seen from formula (4), $s_{1}$ grows with the increase in altitude proportionally to its square, and it depends on angle of viewing area.

While different objects are being searched for, the resolution used is not angular but linear, $d$ (level of

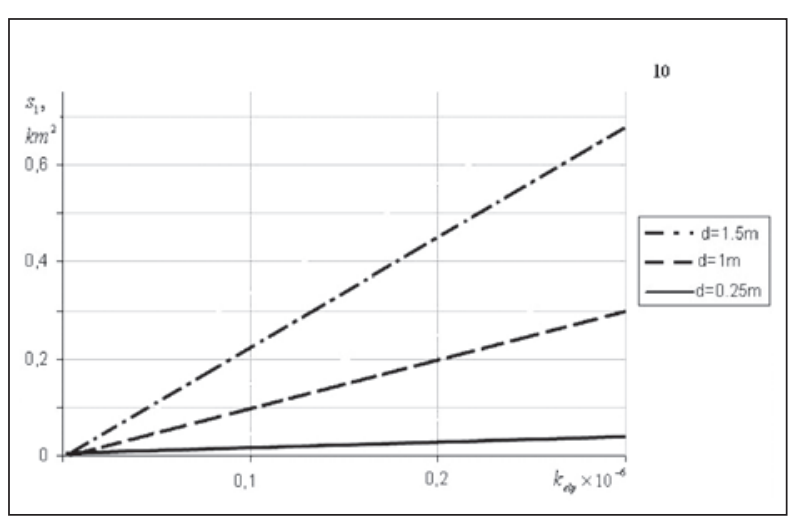

Fig. 4. Dependence of survey region area on the coefficient of the optical system 
detail). This allows the type of object being searched for to be determined. The less level of detail $d$ is, the clearer the picture is on the screen. That is why for the reconnaissance of pinpoint targets, linear resolution of the electrooptical system should be as little as possible (and therefore better). Specialists from the USA have developed the requirements to the level of detail, which should provide recognition of typical objects of survey to some certain level (Review... 2000).

Precision of recognition is characterised by five features.

1. Discovery is the localisation of target being searched for and determination of its coordinates.

2. Common identification is a determination of target class.

3. Precise identification is a determination of target type within a specified class.

4. Descriptive identification is an exposure of configuration and target dimensions and components.

5. Object technical analysis is a determination of affiliation, most probable characteristics, and certain peculiarities.

For instance, if the electrooptical system has linear resolution of $d=4.5 \mathrm{~m}$, then it is possible to determine that the given object looks like an airplane, at $d=1.5 \mathrm{~m}$ it is possible to specify the type of airplane, and at $d=1 \mathrm{~m}$ it is possible to obtain more detailed information about the airplane (its affiliation, purpose, etc.). Thus, value $d$ is defined by a certain reconnaissance task. Analogically to formula (2), it is connected with angular resolution via flight altitude (due to smallness of angular resolution, it is accepted that $\operatorname{tg} \gamma=\gamma[\mathrm{rad}])$

$$
d=H \gamma \text { or } H=d / \gamma \text {. }
$$

From formula (5), it is seen that at constant angular resolution $\gamma$ linear resolution $d$ is connected with altitude by proportional dependence. With an increase in altitude it also increases; image on the screen deteriorates and at some altitude the object will appear to be indistinguishable.

Substituting the value of altitude from (5) to formula (4), we will get the expression for determination of region area

$$
s_{1}=4 d^{2} \frac{\operatorname{tg} \beta_{1} \operatorname{tg} \beta_{2}}{\gamma^{2}}=d^{2} k_{e l p},
$$

where

$$
k_{\text {elp }}=4 \frac{\operatorname{tg} \beta_{1} \operatorname{tg} \beta_{2}}{\gamma^{2}} .
$$

$k_{\text {elp }}$ is a coefficient of the electrooptical system. It combines angles of view and angular resolution of a system, that is it comprehensively characterises the electrooptical system. The better the resolution (less $\gamma$ ) and the greater the angles of system view are, the greater coefficient $k_{\text {elp }}$ will be, and therefore the greater the area that will be surveyed at the given level of object detail $d$.

The graph of dependence of region area $s_{1}$ on the coefficient of $k_{\text {elp }}$ is given in figure 4 .

At constant level of detail $d$, this dependence is linear. The coefficient $k_{\text {elp }}$ changes in quite wide limits. The most significant influence on it is caused by angular resolution of a system. First, it can vary from system to system by time, and second, $k_{\text {elp }}$ is a quadratic function of angular resolution (of quite small value).

Formula (6) is convenient for practical use. For example, let there be the task to discover and precisely identify airplanes on some area $\Delta S$ (if they are present there). For that the level of detail needed is $d=1 \mathrm{~m}$. Then for a certain reconnaissance system, it is possible to calculate the coefficient $k_{\text {elp }}$ according to formula (7) and the value of area $s_{1}$ according to formula (6).

With knowledge of the value of $s_{1}$, the common area of reconnaissance $\Delta S$ and coefficient of its overlapping $\kappa_{\text {ovr }}$, it is possible to calculate the required volume of information in a form of number of needed frames

$$
\Delta n=k_{\mathrm{ovr}} \Delta S / s_{1}=k_{\mathrm{ovr}} B V \Delta t / s_{1}=k_{\mathrm{ovr}} W \Delta t / s_{1},
$$

and average rate of their creation

$$
\dot{n}=\frac{\Delta n}{\Delta t}=k_{\text {ovr }} \frac{W}{s_{1}} .
$$

With the use of value $\dot{n}$, it is possible to calculate the information flow in GBytes/s, which defines the mode of processing.

It is evident that value $\Delta n$ is proportional to the volume of initial information that is necessary to be saved and/or transmitted to the ground (to the control centre or other consumer).

Moreover, with the use of formula (5), it is possible to determine the optimum flight altitude using formula (2) (the width $B$ of the strip covered), to set the speed $V$, to calculate the survey capacity $W=B V$, and calculate the time $t_{t s}=\Delta S / W$ in which the task can be accomplished.

For high quality of initial information processing, the airborne system should have enough memory volume and a high rate of data transmission, and the control centre should have a required number of workplaces for interpreters.

The rate of transmission of frames will depend on:

- the size of the region required to identify the target being searched for in surveyed area $s_{1}$;

- capacity of search $W$.

If we consider $S=k_{\text {ovr }} n s_{1}=$ const, the decrease in $s_{1}$ is inevitably connected with the growth in information volume $n$. The product of $n s_{1}$ shows the main antilogy of reconnaissance, which is a disagreement between the volume of information and the dimensions of the object. Decrease in area $s_{1}$ of surveyed objects will cause a pro- 
portional increase in the number of frames, which will require increase in information flow, volume of memory and airborne memory speed, workplaces for interpretation, etc. It will eventually influence the agility of obtaining the information.

If a panoramic vision system is used for survey, then it is possible to reduce it to a discrete one, presenting $B_{2}$ in the formulas mentioned above via rate of system scanning in the direction of flight. The common area of the strip scanned will also be equal to the product of $n s_{1}$.

\section{Cost of unmanned aeronautical system}

The cost of an unmanned aeronautical system is estimated according to several indices:

- initial cost of a system $C_{0}$;

- operational costs $C_{\text {oper; }}$;

- flight hour cost $C_{\mathrm{fh}}$;

- cost of survey of region $\mathrm{s}_{1}$ of given area $C_{\mathrm{s} 1}$;

- cost of reconnaissance flight $C_{\mathrm{ff}}$.

Initial cost consists of the cost of design and manufacture of the aircraft airframe with all the onboard systems, cost of operational load, and cost of on-ground systems $\left(C_{\mathrm{gr}}\right)$, providing aircraft operation

$$
C_{0}=C_{\text {airfr }}^{m} m_{\text {airfr }}+C_{o p l}^{m} m_{o p l}+C_{g r},
$$

where $\tilde{N}_{\text {airfr }}^{m}$ is the cost of $1 \mathrm{~kg}$ of airframe mass with equipment but without operational load, $C_{o p l}^{m}$ is the cost of $1 \mathrm{~kg}$ of operational load, and $m_{\text {airfr }}$ and $m_{o p l}$ are the masses of the airframe and operational load.

Referring to data given in the journal Avia sojuz, in the USA in 2006 the cost of $1 \mathrm{~kg}$ of UAV airframe was equal to an average of $\$ 3300$, and the cost of operational load was $\$ 17,260$. Besides, it is necessary to mention that over the past few years the mass of operational load has decreased five times faster than airframe mass.

The cost of operation can be estimated as follows

$$
C_{\text {oper }}=C_{\text {oper }}^{t} t_{\text {lif }},
$$

where $C_{\text {airfr }}^{m}$ is the average cost per hour of operation and $t_{\text {lif }}$ is the aircraft service life in hours.

If the assumed (statistical) number of air missions is equal to $N$, then the actual service life can approximately be estimated as $t_{\text {lif }}=N t_{\text {max }}$, where $t_{\max }$ is maximum flight duration.

With these assumptions taken into account, the total cost of an unmanned aeronautical system can be estimated as a sum

$$
C_{\mathrm{UAS}}=C_{0}+C_{\text {oper }}
$$

One of the most important criteria for comparative assessment of unmanned aeronautical systems is cost per flight hour. This can be assessed by the total of initial cost, maximum flight duration $\left(t_{\max }\right)$, and use mul- tiplicity - the number of take-offs $(N)$ - in the following form:

$$
C_{f h}=\frac{C_{0}}{t_{\max } N}+C_{o p e r}^{t} .
$$

The denominator in this formula shows aircraft flight time during an assumed period of operation. It is evident that this index depends on many factors. In particular, in peaceful times it is a sortie rate, and under conditions of combat activities it will decrease depending on the conditions of use and on enemy counteractions. The experience of recent wars demonstrates that the number of air missions for modern unmanned aeronautical systems in conditions of strong counteraction of air defense systems is measured in units or, in the best case, in tens. In this case, when the costs are considered, it is more beneficial to have a comparatively cheap small vehicle. It is assumed that if the cost of the UAV is less than the cost of the target object, then it justifies its purpose.

Now let us determine the cost of the flight of an unmanned aeronautical system. For reconnaissance of some common area $S=B V t$, it is necessary to make $n=$ $S /\left(k_{\text {ovr }} s_{1}\right)$ frames. After all possible influencing factors (operability of equipment, radio visibility, avoidance of enemy air defences, possibility of being hit by antiaircraft fire or enemy aircraft, etc.) are taken into account, if the probability of accomplishing the task is equal to $p_{t s}$, then the cost of the flight is estimated by the value of

$$
C_{f l}=C_{s 1}^{*} n / p_{t s} .
$$

The value $C_{s 1}$ can be determined with the help of flight time and cost per flight hour. During a survey of region $s_{1}$ (Fig. 3), an aircraft passes a distance of $B_{2}=$ $2 H_{2} \operatorname{tg} \beta_{2}=V \Delta t_{1}$. As a result, the flyover time of the given region will be equal to

$$
\Delta t_{1}=\frac{2 H_{2} \operatorname{tg} \beta_{2}}{V} .
$$

Then

$$
C_{s 1}=C_{f h} \Delta t_{1}=C_{f h} \frac{2 H_{2} \operatorname{tg} \beta_{2}}{V} .
$$

It is evident that this index will be one of the most significant. It answers a certain question: what resources should be spent to discover a certain object with $d$ level of detail (for example, a car, an airplane, a ship, etc.) that could be present in region $s_{1}=d^{2} k_{e l p}$.

\section{Agility}

The success of a search mostly depends on time and the quality of the information obtained. The total search time $\left(t_{t s t}\right)$ consists of the time necessary to fulfil separate stages:

- time needed for UAV pre-flight maintenance $\left(t_{p f}\right)$;

- time needed to fly to the region being searched $\left(t_{\text {reg }}\right)$;

- time needed to accomplish the search $\left(t_{s a c}\right)$; 
- time needed to process and store data $\left(t_{\text {stor }}\right)$ (if required);

- time needed to transmit data to the control centre $\left(t_{\text {trans }}\right)$;

- time needed to process data and transmit it to the consumer $\left(t_{\text {proces }}\right)$

$t_{t s t}=t_{p f l}+t_{\text {reg }}+t_{\text {sac }}+t_{\text {stor }}+t_{\text {trans }}+t_{\text {proces }}$.

The value $t$ characterises the agility of obtaining information from the UAV.

The time needed for pre-flight maintenance $t_{p f l}$ depends on the rate of preparedness for the flight.

The time needed to fly to the region being searched can approximately be calculated as $t_{\text {reg }}=L / V+\Delta$, where $L$ is the distance between the search area and place of takeoff and $V$ is flight speed, which can be chosen depending on the task: either maximum or cruising. $\Delta(2-3$ minutes) is a correction to the time for acceleration of the aircraft and climb to a specified altitude.

The time necessary to accomplish the search $t_{s a c}$ can be determined in two ways:

- in the process of setting the route and the flight profile by means of carrying out engineering-navigational calculation (Silkov 1997); in the process of setting the area of reconnaissance using the formula (3) $t_{s a c}=S / W$. If the search is being carried out with overlapping territory, then the quotient obtained is multiplied by the overlapping coefficient. Usually it is within the range of $k_{\text {ovr }}=1.1 \ldots 1.15$.

In case of flight along a random route, the reconnaissance time is determined by the fuel reserve $t_{s a c}=$ $m_{\text {fuel }} / q_{\mathrm{h}}$, where $m_{\text {fuel }}$ is a reserve of fuel for the accomplishment of the task (excluding all regulated residuals) and $q_{\mathrm{h}}$ is fuel consumption per hour.

All the other components of time $\left(t_{p r}+t_{t r}+t_{c o n}\right)$ and their values depend on certain systems of information acquisition, storage and processing.

If during flight there was a task to determine objects in some area but not survey a specific zone, then we can calculate the total area relative to $S=n s 1$ in the specific zone.

Therefore, the criterion of cost $C_{s 1}$ will be the same for discrete and panoramic frames.

The productivity-cost-efficiency complex of factors was developed to assess the effectiveness of the observation and searching abilities of unmanned aeronautical systems. These factors can also be used to comparatively assess several types of UAVs. For that they calculate specified indices for each type and then, for example, applying hierarchy analysis technique they determine comprehensive estimates for each of them and choose the best sample UAV (Saaty 1992).

\section{Conclusions}

1. A complex of factors, productivity - cost - efficiency, was developed to assess the effectiveness of the observation and searching abilities of unmanned aeronautical systems.

2. To assess the technical capabilities of optoelectronic systems, an integrated indicator, which includes look-up angles and angular discrimination of the camera, was proposed.

3. It is shown that one of the most important parameters determining the time of filming a preset area, the volume of information being transferred and the speed at which this is done, is a reference area that depends on survey instrument data and on the required level of detail of objects on the ground.

4. The methodological approach that was developed can be used for a comparative evaluation of unmanned aeronautical systems and for the substantiation of decisions that need to be made while searching for specific objects.

\section{References}

Goraj, Z. 2003. Civilian and military unmanned aerial vehicles - overview of European and American effort and challenges for the future, Aviation 7(1): 3-15.

Mitrakovich, A.; Samkov, A.; Silkov, V. 2009. Unmanned Aerial Vehicle: the Techniques of Approximate Calculations of Key Parameters and Characteristics. Kiev: CNII VVT BS Ukrainy. 302 p.

Rastopchin, V. 2012. Elementary evaluation principles of the application efficiency of unmanned aeronautical systems for aerial reconnaissance [online]. Available from Internet: www.avia.ru.

Review of ONR's Uninhabited Combat Air Vehicles Program Commission on Physical Sciences, Mathematics and Applications. 2000.

Saaty, T. L. 1992. The Hierarchon: A Dictionary of Hierarchies. Pennsylvania, Pittsburgh: RWS Publications. 496 p. ISBN 0-9620317-5-5

Silkov, V. 1997. Aircraft Flight Dynamics. Kiev: KMU GA. 425 p.

Silkov, V. 2004. Military Maneuvering of Aircraft. Kiev: NAOU. $340 \mathrm{p}$. 